\title{
The effect of heat conduction on the interaction of disk and corona around black holes
}

\author{
E. Meyer-Hofmeister and F. Meyer
}

Max-Planck-Institut für Astrophysik, Karl-Schwarzschildstr. 1, 85740 Garching, Germany

e-mail: emm@mpa-garching.mpg.de

Received 5 August 2005 / Accepted 9 November 2005

\begin{abstract}
Heat conduction plays an important role in the balance between heating and cooling in many astrophysical objects, e.g. cooling flows in clusters of galaxies. Here we investigate the effect of heat conduction on the interaction between a cool disk and a hot corona around black holes. Using the one-radial-zone approximation, we study the vertical structure of the disk corona and derive evaporation and coronal mass flow rates for various reduced thermal conductivities. We find lower evaporation rates and a shift in the evaporation maxima to smaller radii. This implies that the spectral state transition occurs at a lower mass flow rate and a disk truncation closer to the black hole. Reductions of thermal conductivity are thought to be magnetically caused and might vary from object to object by a different configuration of the magnetic fields.
\end{abstract}

Key words. accretion, accretion disks - black hole physics - X-rays: binaries - cooling flows

\section{Introduction}

Heating by thermal conduction is an important process in many astrophysical objects. The balance between cooling and heating processes determines the structure of hot matter in contact with cooler regions. For the case of a dwarf nova accretion disk, we investigated this interaction (Meyer \& Meyer-Hofmeister 1994) and found that a "siphon flow" leads to evaporation of the disk. The same happens in disks around neutron stars and in both galactic and supermassive black holes (Meyer-Hofmeister $\&$ Meyer 1999; Meyer et al. 2000). The heat conduction is an essential element; otherwise the coronal gas would not lose energy, but would instead become hotter and reach virial temperatures as it is present in advection-dominated flows.

Recently the same physical process was discussed by several authors in connection with the cooling flow problem for the hot intra-cluster medium (ICM). The work of Medveden \& Narayan (2001) focuses on the question as to what degree chaotic magnetic fields suppress conduction relative to the Spitzer level. They find that thermal conduction in a weakly collisional plasma with turbulent magnetic fields approaches the Spitzer limit. Zakamska \& Narayan (2003) find from the investigation of five galaxy clusters that the Spitzer formal with a conduction coefficient reduced to about $30 \%$ gives a good description of the observed radial profiles of electron density and temperature. Ghizzardi et al. (2004) discuss which fraction of the Spitzer value would be appropriate xplaining the Virgo/M 87 observations. Voigt \& Fabian (2004), on the other hand, found support for an unhindered heat conduction from their analysis of 16 galaxy clusters using Chandra data. One third of the Spitzer value was used in hydrodynamic cosmological simulations of galaxy clusters by Dolag et al. (2004). In this context Soker et al. (2004) suggest a heat conduction along magnetic field lines. Okabe \& Hattori (2004) suggest a suppression of heat conduction by magnetic fields generated most strongly in the direction perpendicular to the temperature gradient. All these results point to a reduced heat conduction in many sources.

In the original context of disk/corona interaction, the effect of reduced thermal conduction has already been considered by a scaling procedure (Meyer et al. 2000). In the present work we evaluate the effect of heat conduction on the evaporation of accretion disks in detail. Connected with the evaporation efficiency, this means a possible change in the resulting truncation radius. Motivation also comes from the fact that new computations including the irradiation of the coronal gas from the inner region (Meyer-Hofmeister et al. 2005 (hereafter MLM05); Liu et al. 2005) have led to radii that seem larger than indicated by observations (Yuan \& Narayan 2004). In these works the thermal conduction was taken according to the standard value derived by Spitzer (1962). The question then arises whether a reduced heat conduction could be present in the disks around black holes. Here we study the effect of heat conduction on evaporation and the truncation of the accretion disk.

In Sect. 2 we give a short description of the accretion geometry and the interaction of corona and disk. In Sect. 3 we 
present the results for reduced heat conduction, and a discussion of the consequences and conclusions follow in Sect. 4.

\section{The physics of a hot corona above a cool disk}

In the work by Meyer et al. (2000), the interaction of disk and corona was approximated by a one-zone model that allows us to evaluate the evaporation of mass from the cool disk into a coronal flow above the disk. The basic process is the following. The coronal temperature is kept up by friction that releases gravitational energy from accretion. The temperature gradient between the corona and cool disk causes a heat flow. The density in the corona adjusts itself by evaporation of gas from the disk so that this heat flow is balanced by evaporation, radiation, and advection. This equilibrium density in the corona implies a coronal mass accretion rate that is fed by gas from the disk and determines the disk evaporation rate.

The five equations describing the process are: (1) the equation of continuity, (2) the $z$-component of momentum equation, (3) and (4) the two energy equations for ions and electrons, and (5) the equation for the thermal conduction for a fully ionized plasma (see Liu et al. 2002). The effect of Compton cooling and heating of coronal electrons by photons from the central area was recently worked out in more detail and found to be important for the evaporation process (MLM05). The five dependent variables are pressure, ion and electron temperature, vertical mass flow, and heat flux. The boundary conditions are taken (a) at the bottom of the corona, temperatures given, no heat inflow, and (b) at some height (free boundary), sound transition, no influx of heat.

The general picture is as follows. Controlled by the interaction of the disk and corona, gas evaporates from the disk into the corona and flows inward in the form of a hot advectiondominated flow. The mass flow rate in the thin disk is thereby diminished. The evaporation rate increases with decreasing distance from the compact object, but reaches a maximum at a certain distance $R_{\text {evmax }}$ from the black hole. The balance between this maximum rate and the mass flow rate in the disk determines the mode of accretion in the inner region. Only if the mass flow rate in the outer disk is higher than this maximum value of the evaporation rate, the disk "survives" this reduction in mass flow and continues inward. Otherwise, for lower mass flow rates in the disk, the disk becomes truncated at a certain distance (where all mass is evaporated). From then on all mass flows inward via the coronal/ADAF flow. This is the same picture as the configuration of accretion flow in different spectral states derived by Esin et al. (1997) in connection with the application of the scheme of advection-dominated accretion to Nova Muscae 1991.

The agreement between the results of the one-zone approximation and observed features seems to confirm that our model describes the qualitative picture of the change from accretion via a disk to the advection-dominated flow (ADAF). Such agreement was found for $\mathrm{X}$-ray transient sources in connection with the spectral transitions (Meyer et al. 2000), especially the hysteresis in the transition luminosity (MLM05), as well as accretion disk evolution (Meyer-Hofmeister \& Meyer 1999), and also in connection with the truncated disks in low-luminosity
AGN (Liu \& Meyer-Hofmeister 2001). But if we want to extract detailed qualitative results, we have to keep in mind that a number of simplifications used in the description lead to uncertainties of the computational results. Two free parameters (for given mass of the central object and chemical abundance) enter into the evaluation of the evaporation rate: for the viscosity parameter $\alpha$, we use the value 0.3 suggested by observations (for a discussion see MLM05). Note that in our one-zone calculations, $\alpha$ parameterizes frictional heat release and also radial flow of mass and angular momentum. Its value is not directly comparable to the value of the standard Shakura and Sunyaev $\alpha$ value (Shakura \& Sunyaev 1973). For a comparison with the ratio of stress to pressure in magneto-hydrodynamic calculations, see a discussion by Hawley \& Krolik (2001). The second parameter in the evaluation of the evaporation rate is the thermal conduction.

From our recent work on hysteresis in the spectral state transitions, it became obvious that the irradiation of the corona has an important effect on evaporation rates and truncation of the cool accretion disk. We denote by $R_{\text {evmax }}$ the shortest distance at which truncation still can occur. Earlier computations without including the irradiation (Meyer et al. 2000) had led to a distance of a few hundred Schwarzschild radii. Including the irradiation of the corona, we now obtain values about 2.5 times larger. This is the innermost disk truncation; for lower accretion rates the truncation lies farther outward, while for higher rates the disk is not truncated anymore. How do these radii compare with radii found from observations? The observed radii should be equal or larger than $R_{\text {evmax }}$, depending on the mass flow rate in the disk. Despite the fact that one should be careful with such a comparison and not take signatures of a reflecting component as the disk truncation, the observations point to lower values. A compilation of radii in the paper of Yuan \& Narayan (2004, Fig. 3) suggests values around 100 Schwarzschild radii. Esin et al. (2001) use the multiwavelength observations of the X-ray nova XTE J1118+480 to constrain the accretion geometry and find a disk truncation in outburst at $\geq 55$ Schwarzschild radii. The question arises whether a reduced thermal conduction could be present in the accretion disks around black holes and how this would change the evaporation process. In the next section we show our results for reduced heat conduction.

\section{Computational results}

We take a black hole mass of $M=6 M_{\odot}$. Results can be scaled for other masses. We, therefore, show the evaporation rates measured in Eddington accretion rate $\dot{M}_{\text {Edd }}=L_{\text {Edd }} / 0.1 c^{2}$ with $L_{\mathrm{Edd}}=4 \pi G M c / \kappa_{\mathrm{es}}, \kappa_{\mathrm{es}}$ electron scattering opacity. The distances are measured in Schwarzschild radii $R_{\mathrm{S}}=2 G M / c^{2}$. We use the computer code as described in recent work (LMM05). The heat conduction coefficient $\kappa_{0}$

$\kappa_{0}=10^{-6} \frac{\mathrm{g} \mathrm{cm}}{\mathrm{s}^{3} \mathrm{~K}^{7 / 2}}$

enters the equation for the heat flux

$F_{\mathrm{c}}=-\kappa_{0} T^{5 / 2} \mathrm{~d} T / \mathrm{d} z$ 
with $T$ temperature, and $z$ height above midplane. For our calculations we take a fraction $\lambda$ of the standard value for a fully ionized plasma (Spitzer 1962).

The heat conduction also enters in our lower boundary condition at the bottom of the corona, and, without going into detail, this results in a change of the relation between pressure and heat flux (Meyer et al. 2000, Eq. (54)) to $P=A \cdot F_{\mathrm{c}} \lambda^{-1 / 2}$ (compare also Liu et al. 1995).

For taking into account Compton cooling and heating by radiation from the innermost region we use $L=\eta \dot{M} c^{2}, \eta=0.1$ for the luminosity of the central source for various heights of the corona along with $\eta=0.1$ with $\dot{M}$ central mass accretion rate. We calculate the irradiation flux from the central region as in recent work (MLM05, Liu et al. 2005).

\subsection{The evaluation of evaporation rates consistent with irradiation from the central region}

We calculate the evaporation rates for a series of distances from the black hole. We consider both cases of irradiation of the corona: (1) hard radiation from an inner region filled with a hot coronal gas (advection-dominated accretion flow, ADAF, or (2) soft radiation from an accretion disk in the inner region. For our analysis of the hard state we take a mean photon energy of $100 \mathrm{keV}$ for the radiation from the central source. We are mainly interested in case (1) since this describes the situation when the disk is truncated. The truncation radius depends on the mass flow rate in the cool disk. This is the situation as long as the system is in the hard state. Case (2) is relevant here only at the moment when the mass flow rate decreases from a rate above the maximal evaporation rate (the disk then extends inward to the last stable orbit) to a rate below the maximal evaporation rate, and the disk breaks up at the distance $R_{\text {evmax }}$.

The evaporation rate is a function of the distance and of the radiation from the central region, if the effect of irradiation is included. In the one-zone approximation, the mass flow entering and leaving is modeled appropriately for a corona at the inner disk edge, i.e. the mass flow rate in the disk equals the evaporation rate. As this mass flow continues into the central region it causes the radiation which then irradiates the corona at the truncation radius. In other words, the adequate irradiation to be included corresponds to the evaporation rate itself. Therefore an iterative procedure is necessary to determine the evaporation rate consistent with the irradiation; evaporation rates are evaluated for different values of radiation from the inner region until we find the evaporation rate consistent with the irradiation produced by exactly a mass flow rate equal to the evaporation rate.

In recent work we included the irradiation effect (MLM05, Liu et al. 2005) only for the maximal evaporation rates at $R_{\text {evmax }}$, which is important for the spectral transition. For the present investigation we now have determined the rates all all distances consistent with the irradiation effect. Figure 1 thus displays a sequence of disk truncation radii. These results then also allow us to discuss the slope of the evaporation rate distance relation.

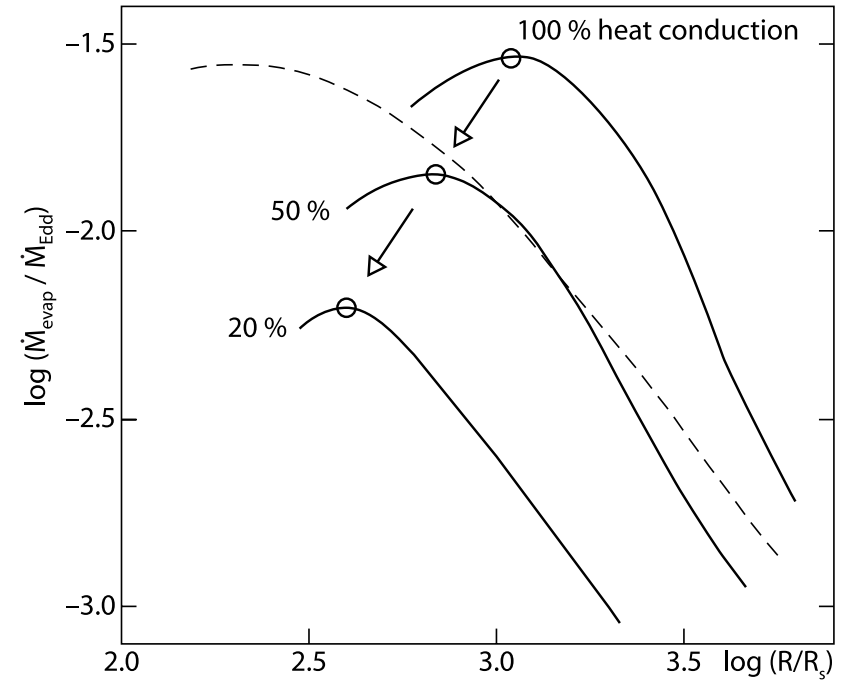

Fig. 1. Effect of reduced heat conduction: evaporation rate as a function of distance from the black hole (hard spectral state). Solid lines: thermal conduction according to standard Spitzer rate and reduced to $50 \%$ and $20 \%$. Dashed line: $100 \%$ heat conduction, but without irradiation. Reduced heat conduction leads to disk truncation farther inward. The maxima give locations of innermost disk truncation at spectral transition.

In Fig. 1 we also show our new results for the reduced heat conduction. We evaluated evaporation rates for the standard Spitzer value heat conduction and a reduction to $50 \%$ and $20 \%$ of this value. As already pointed out in the earlier work, in the hard state the electron energy decreases with increasing distance, the Compton cooling from the central source turns into heating, and the irradiation yields higher evaporation rates than without irradiation.

\subsection{The evaporation rates for hard irradiation}

As shown in Fig. 1, reduced heat conduction strongly affects both the evaporation and the location of the maxima. For example if one assumed a mass flow of $0.005 \dot{M}_{\text {Edd }}$ from the outer disk with $100 \%$ of the Spitzer value of heat conduction, the disk would be truncated at about 3850 Schwarzschild radii. But with a heat conduction reduced to 50 and $20 \%$, respectively, the truncation moves in to 1860 and $570 R_{\mathrm{S}}$, a factor of almost 7 closer to the center in the latter case. For low mass accretion rates when the disk truncation is at large distances, the irradiation effect becomes small. In Fig. 1 we show for comparison results for $100 \%$ heat conduction without irradiation (MLM05). It can be seen that the curves for $100 \%$ heat conduction with and without irradiation begin to converge at large distances.

Interesting is the shift of the evaporation maxima in distance, from about $1100 R_{\mathrm{S}}$ to 700 and $400 R_{\mathrm{S}}$ for reduced heat conduction. Also the amplitude of the maxima is lowered. For a heat conduction reduced to $20 \%$ of the standard Spitzer value, we find an innermost disk truncation at $R_{\mathrm{S}}$ corresponding to a mass flow rate of $0.006 \dot{M}_{\text {Edd }}$. This rate can be compared with the results of Maccarone (2003), who critically analyzes observations to deduce the luminosity of neutron star and black hole 


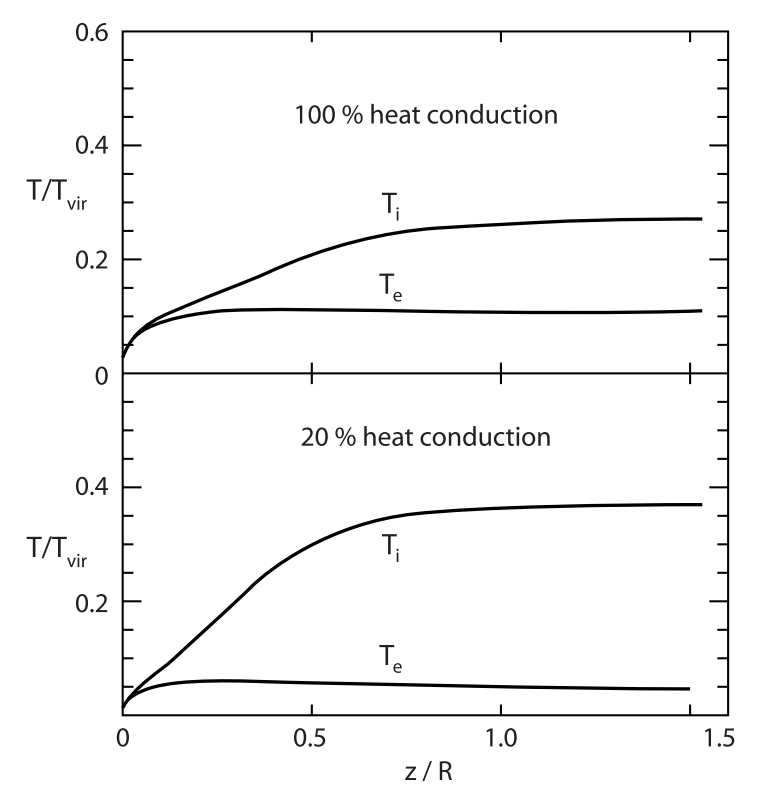

Fig. 2. Coronal temperatures: electron and ion temperatures (measured in virial temperature) at height $z / R, R$ distance from black hole, $z$ height above midplane.

transient binaries at the soft/hard spectral transition. Our value derived for reduced heat conduction is relatively low, but lies in the range found from the observations.

\subsection{The coronal structure for hard irradiation}

Reduced heat conduction changes the vertical structure of the corona. We compare the structure at peak evaporation rate for the two cases: heat conduction either equal to the standard Spitzer value or reduced to $20 \%$ (compare Fig. 1). In Fig. 2 we show the electron and ion temperature as a function of $z / R$. Temperatures are measured in virial temperature $T_{\text {vir }}=G M R \cdot \mu / \mathfrak{R}$. There is a clear change with heat conduction. For the reduced value, the electron temperature is lower and the ion temperature is higher than for the more effective heat conduction. Note that the virial temperatures depend on the distance from the black hole, which is different in the two cases. The corresponding values of pressure at the lower boundary of the corona are $\log P=7.04 \mathrm{~g} \mathrm{~cm}^{-1} \mathrm{~s}^{-2}$ (100\% heat conduction) and $7.53 \mathrm{~g} \mathrm{~cm}^{-1} \mathrm{~s}^{-2}(20 \%)$, which is a factor of 3 higher in the latter case.

\subsection{Computational results for soft irradiation}

The effect of soft irradiation is only of interest here at the moment the disk becomes truncated by evaporation, i.e. the soft/hard transition. Compton cooling leads to lower evaporation rates (MLM05). Including reduced heat conduction decreases the evaporation rates even more. In our computations we encountered problems finding solutions for the coronal structure to fulfill our upper boundary conditions such as sound transition and no heat inflow from infinity. Further work is needed to clarify whether the condition $P \rightarrow 0$ for large $z$ can be achieved with a subsonic structure.
For heat conduction reduced by a factor of 5 and soft irradiation, we found the maximal evaporation rate of $\dot{M}_{\text {evap }} / \dot{M}_{\text {Edd }}=$ 0.004 at about 400 Schwarzschild radii. The difference between the rates for hard and soft irradiation allows us to understand the hysteresis in the transition luminosity of X-ray transients. For reduced heat conduction the difference becomes small, less than a factor of 2, and much less than what was found for unreduced heat conduction. This might point to a problem understanding the hysteresis seen in X-ray nova outburst light curves for the case of strongly reduced heat conduction; if not other effects enlarge this difference. Such further effects might be due to time delays in the transition and recondensation of hot coronal gas into the disk. We are presently investigating this situation (Liu \& Meyer).

\section{Discussion}

\subsection{Disk truncation radius as a function of the mass accretion rate}

The curves in Fig. 1 show both the dependence of evaporation rates on the distance and the disk truncation for a given mass flow rate in the disk. It might be interesting to analyze observations of X-ray transients to see how the inner disk edge moves inward or outward as a result of increasing or decreasing mass flow.

Our theoretical analysis only allows us to make rough predictions due to the uncertainties in the approximations. The curves in Fig. 1 are not straight lines. For standard heat conduction and for a range of distances of $R_{\mathrm{tr}} / R_{\mathrm{S}}$ from $10^{3.3}$ to $10^{3.9} \mathrm{~cm}$ we find a slope of

$\Delta \log R_{\mathrm{tr}} / \Delta \log \dot{M} \approx-0.5$.

We find a slightly steeper slope of about -0.8 for a reduction to $20 \%$ (for distances $R_{\mathrm{tr}} / R_{\mathrm{S}}$ from $10^{2.8}$ to $10^{3.3} \mathrm{~cm}$ ).

If we compare our results with changes of the inner disk location of the X-ray transient source XTE J1118, in particular we find for truncation radii and luminosities the following values in the literature: in outburst $\log R_{\mathrm{tr}} / \log R_{\mathrm{S}}=1.74$ for $\log L / L_{\mathrm{Edd}}=-1.7$, (Esin et al. 2001) and in quiescence $\log R_{\mathrm{tr}} / \log R_{\mathrm{S}} \approx 4$ for $\log L / L_{\mathrm{Edd}}=-8.5$ (McClintock et al. 2003). Note that the source is also in the hard spectral state in outburst. This gives the value 0.33 for $\Delta \log R_{\mathrm{tr}} / \Delta \log \dot{L}$. If we want to translate this into a radius-mass accretion rate relation, we need to keep in mind that for low mass accretion rates, radiation processes scale with particle density squared, thus leading roughly to a scaling of the luminosity with $\dot{M}^{2}$. This would yield an approximate agreement between the theoretical model and observations.

A relation between truncation radius and luminosity for various sources from bright AGN and X-ray binaries to dim objects as $\mathrm{Sgr} \mathrm{A} *$ is shown in the investigation of X-ray bright, optically normal galaxies by Yuan \& Narayan (2004, Fig. 3)

\subsection{Is heat conduction always the same?}

A number of discussions have arisen around cooling flows and magnetic fields. We note that in the more general frame of 
condensation and evaporation between corona and cool disk evaporation, flows can straighten out fields from the cool gas as they carry the fields with them. It seems conceivable that the value of the thermal conduction varies from object to object and also in one object with time depending on the accretion flow history. Zdziarski et al. (2004) study the long-term variability of the X-ray nova GX 339-4 and discuss the spectral changes in different outbursts. Especially during the two recent outbursts, the hard/soft transition in the second one occurred at a higher luminosity than that in the preceding one. They argued that this difference could arise from the different accretion disk history. The magnetic field pattern in the disk might be influenced by this history of more or less mass flow in the disk.

\section{Conclusions}

We have evaluated how a reduced heat conduction affects the evaporation of gas from a cool disk to a hot coronal flow/ADAF. The physical situation of evaporation (or condensation) between hot ("coronal") and cool ("disk") gas is the same around stellar black holes and in galaxies and clusters of galaxies. For clusters of galaxies, reduced heat conduction seems to be supported by observations in several cases.

In our theoretical modeling, evaporation rates become lower with reduced heat conduction, and the location where the evaporation efficiency reaches its maximum moves inward by a factor of 7 for the reduction to $20 \%$. This moves the truncation radii closer to an agreement with observations, but a significant difference exists still. On the other hand, the change of truncation radii with accretion rate $\Delta \log R_{\mathrm{tr}} / \Delta \log \dot{M}$ in our results is in reasonable agreement with the numbers derived from observations for galactic and supermassive black holes.

Interesting is the strong dependence of spectral transition on heat conduction. A further reduction might arise from a different magnetic field situation. This can affect the transition from a very bright state to a very dim state in the AGN of elliptical galaxies as suggested by Churasov et al. (2005).

\section{References}

Churazov, E., Sazonov, S., Sunyaev, R., et al. 2005, MNRAS, 363, L91

Dolag, K., Jubelgas, M., Springel, V., et al. 2004, ApJ, 606, 97

Esin, A. A., McClintock, J. E., \& Narayan, R. 1997, ApJ, 489, 865

Esin, A. A., McClintock, J. E., Drake, J. J., et al. 2001, ApJ, 505, 854

Ghizzardi, S., Molendi, S., Fabio, P. M., et al. 2004, ApJ, 609, 638

Hawley, J. F., \& Krolik, J. H. 2001, ApJ, 548, 348

Liu, B. F., \& Meyer-Hofmeister, E. 2001, A\&A, 372, 386

Liu, B. F., Mineshige, S., Meyer, F., et al. 2002, ApJ, 575, 117

Liu, B. F., Meyer, F., \& Meyer-Hofmeister 2005, A\&A, 442, 555

Liu, F. K., Meyer, F., \& Meyer-Hofmeister, E. 1995, A\&A, 300, 823

Maccarone, T. J. 2003, A\&A, 409, 697

McClintock, J. E., Narayan, R., Garcia, M. R., et al. 2003, ApJ, 593, 435

Meyer, F., \& Meyer-Hofmeister, E. 1994, A\&A, 288, 175

Meyer-Hofmeister, E., \& Meyer, F. 1999, A\&A, 348, 154739

Meyer-Hofmeister, E., Liu, B. F., \& Meyer, F. 2005, A\&A, 432, 181 (MLM05)

Meyer, F., Liu, B. F., \& Meyer-Hofmeister, E. 2000, A\&A, 361, 175

Narayan, R., \& Medveden, M. V. 2001, ApJ, 562, L129

Okabe, N., \& Hattori, N. 2004, In Proc. of The Riddle of Cooling Flows in Galaxies and Clusters of Galaxies, Charlottesville 2003, ed. T. Reiprich, J. Kempner, \& N. Soker, http://www . astro.virginia.edu/coolflow/

Shakura, N. I., \& Sunyaev, R. A. 1973, A\&A, 24, 337

Spitzer, L. 1962, Physics of Fully Ionized Gases, 2nd edition (New York, London: Interscience Publ.)

Soker, N., Blanton, E. L., \& Sarazin, C. L. 2004, A\&A, 422, 445

Voigt, L. M., \& Fabian, A. C. 2004, MNRAS, 347, 1130

Yuan, F., \& Narayan, R. 2004, ApJ, 612, 724

Zakamska, N. L., \& Narayan, R. 2003, ApJ, 582, 162

Zdziarski, A. A., Gierliński, M., Mikolajewska, J., et al. 2004, MNRAS, 351, 791 